\title{
Neutrophil count is associated with myeloid derived suppressor cell level and presents prognostic value for hepatocellular carcinoma patients
}

\author{
Xing Li ${ }^{1,2,3}$, Yan-Fang Xing ${ }^{4}$, Ai-Hua Lei ${ }^{1,2}$, Qiang Xiao ${ }^{1,2}$, Zhi-Huan Lin ${ }^{3}$, Ying-Fen \\ Hong $^{3}$, Xiang-Yuan $\mathbf{W u}^{3}$, Jie Zhou ${ }^{1,2}$ \\ ${ }^{1}$ Program in Immunology, Affiliated Guangzhou Women and Children's Medical Center, Zhongshan School of Medicine, Sun \\ Yat-Sen University, Guangzhou, China \\ ${ }^{2}$ Institute of Human Virology, Zhongshan School of Medicine, Sun Yat-Sen University, Guangzhou, China \\ ${ }^{3}$ Department of Medical Oncology and Guangdong Key Laboratory of Liver Disease, the Third Affiliated Hospital of Sun Yat-Sen \\ University, Guangzhou, China \\ ${ }^{4}$ Department of Nephrology, the Third Affiliated Hospital of Guangzhou Medical University, Guangzhou, China \\ Correspondence to: Xiang-Yuan Wu, email: wuxiangy@mail.sysu.edu.cn \\ Jie Zhou, email: zhouj72@mail.sysu.edu.cn \\ Keywords: neutrophil counts, hepatocellular carcinoma, patient selection, prognosis, myeloid derived suppressor cell
}

Received: October 07, 2016

Accepted: February 06, 2017

Published: February 17, 2017

Copyright: Li et al. This is an open-access article distributed under the terms of the Creative Commons Attribution License (CC-BY), which permits unrestricted use, distribution, and reproduction in any medium, provided the original author and source are credited.

\section{ABSTRACT}

Myeloid Derived Suppressor Cell (MDSC) has been raised to be a novel target for multiple cancers. However, target agents on MDSC have not display promising efficacy. One of the critical reasons shall be less optimal patient selection. In the present study, we aimed to identify clinical parameters relevant to MDSC level in hepatocellular carcinoma (HCC) patients for future MDSC targeted therapy. In the present study, a series of 55 HCC patients (testing group) and 20 healthy donors were analyzed investigating frequencies of MDSC in peripheral blood mononuclear cells (PBMC). As a result, we found that MDSC level was increased in HCC patients compared to healthy donors $(10.33 \%$ vs $1.54 \%, p<0.0001)$. The monocytes $\left(r^{2}=0.2875\right.$, $p<0.0001)$, neutrophils $\left(r^{2}=0.3630, p<0.0001\right)$ and platelet counts $\left(r^{2}=0.0828\right.$, $p=0.0331$ ) in circulation was positively associated with MDSC level. Then, the prognostic value of the above predictors was determined in a retrospective database of 255 HCC patients (validation group). The baseline characteristics of testing and validation group were similar. Multivariate analysis by Cox regression revealed that neutrophil count was an independent predictor for overall survival (OS) $(p=0.000$, HR 1.065, 95\% CI 1.028-1.103), with the rest parameters failed to reach a significant result. In summary, the present study firstly identified blood neutrophil counts was a predictor of MDSC level in PBMC for HCC patients. And, patients with higher neutrophil count level might be the optimal patient subgroup for MDSC targeted therapy.

\section{INTRODUCTION}

Myeloid Derived Suppressor Cell (MDSC) has been raised to be a novel target and a prognostic factor for many malignant diseases, especially for hepatocellular carcinoma (HCC) [1-6]. However, target agents on MDSC have not display promising efficacy for HCC patients. One of the critical reasons shall be the heterogeneity of patients which made patient selection very complicated for the success of clinical trials. However, there is no investigation on the patient selection criteria for MDSC based clinical trials. Directly testing MDSC level in HCC patients might be a plausible way to select optimal patients. However, it is not easy to standardize procedure and MDSC testing among different researchers and institutions [7], which make multicenter clinical trials less practical. Thus, it is necessary to identify clinically practical parameters as specific patient selection criteria.

Neutrophil, monocyte and platelet count in blood was reported to be promising prognostic factors for $\mathrm{HCC}$ 
patients by a series of studies including ours [8-11], which made them potential patient selection criteria for clinical trials. However, the mechanism was not identified yet. Interestingly, neutrophils in blood consisted of neutrophils and MDSC $[12,13]$. Thus, neutrophils count might reflect the MDSC level of HCC patients and be a practical selection criterion for multicenter clinical trials.

In the present study, we investigated the circulation MDSC levels and their association with clinical parameters of peripheral blood cell counts. Finally, the prognostic value of MDSC related clinical parameters were tested by integrated into CLIP score system. The present study aimed to identify a promising patient selection criterion for MDSC targeted treatments.

\section{RESULTS}

\section{PMN-MDSCs and M-MDSCs level were elevated in HCC patients}

A total of $55 \mathrm{HCC}$ patients and 20 aged and sex match controls were tested PMN-MDSC (HLA-DR-/low

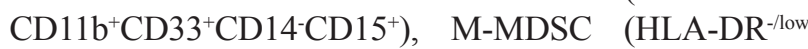

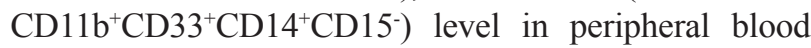
mononuclear cells (PBMCs) [13] Figure 1A. PMN-MDSCs were $\mathrm{CD}_{66 \mathrm{~b}^{+}}$and M-MDSCs were CD66b. (Figure 1B). As a result, we found that total MDSC level in HCC patients (median $10.33 \%$, range $1.43-35.34 \%$ ) were significantly increased compared with health control (median 1.54\%, range 0.91-3.76\%). PMN-MDSC level (median 6.74\%, range $0.60-26.03 \%$ ) was increased in $\mathrm{HCC}$ patients compared to healthy donors (median $1.35 \%$, range 0.40 $3.13 \%$ ). M-MDSC level was also higher in HCC group (median $2.79 \%$, range $0.00-13.99 \%$ ) than the healthy control (median $0.44 \%$, range $0.00-1.48 \%$ ). (Figure $1 \mathrm{C}$ ).

MDSCs in tumor were relatively higher than PBMC. And, the results were parallel between PMN-MDSC and M-MDSC (Figure 1D). Notably, MDSC and PMN-MDSC levels were positively related to those in PBMC (Figure 1E), with M-MDSC levels reached a marginal positive result.

In order to confirm immune suppressive capacity of PMN-MDSC/M-MDSC in HCC patients, T cells and PMN-MDSC or M-MDSC were purified from PBMC using Flow sorting, respectively. CFSE-labeled PBMCderived $\mathrm{CD} 3+\mathrm{T}$ cells were stimulated with anti-CD3/ anti-CD28 with or without PMN-MDSC or M-MDSC. CD4+ and CD8+ $\mathrm{T}$ cell proliferation were abrogated by the addition of PMN-MDSC or M-MDSC with a dosage dependent manner. (Figure 2).

\section{PMN-MDSCs and M-MDSCs levels were associated with multiple parameters of peripheral blood cell counts}

The association between clinical parameters of peripheral blood cell counts and MDSC level were tested by Linear regression. MDSC levels were associated with monocytes $\left(\mathrm{r}^{2}=0.2875, p<0.0001\right)$, neutrophils $\left(\mathrm{r}^{2}=0.3630, p<0.0001\right)$ and platelet counts $\left(\mathrm{r}^{2}=0.0828\right.$, $p=0.0331)$ in circulation significantly. Specifically, PMN-MDSC levels positively related to monocytes $\left(\mathrm{r}^{2}=\right.$ $0.3240, p<0.0001)$, neutrophils $\left(\mathrm{r}^{2}=0.3803, p<0.0001\right)$ and platelet counts $\left(\mathrm{r}^{2}=0.0963, p=0.0211\right)$ in circulation significantly. Similarly, M-MDSC levels displayed positive association with monocytes $\left(\mathrm{r}^{2}=0.1355, p=0.0057\right)$ and neutrophils $\left(\mathrm{r}^{2}=0.2145, p=0.0004\right)$. (Figure 3 )

\section{Prognostic value of clinical parameters related to MDSC levels}

In order to determine the prognostic value of MDSC related clinical parameters, a retrospective consisted of 245 HCC patients were utilized. The baseline characteristics of the validation group were similar to the testing group (Table 1). The prognostic value for overall survival (OS) of the clinical parameters related to MDSC were tested by integrated into Cancer of the Liver Italian Program (CLIP) score system, a well acknowledged prognostic score system for HCC patients [8, 10, 14]. Multivariate analysis by Cox regression revealed that neutrophils were associated with unfavorable OS $(p=0.000$, HR 1.065 , 95\% CI 1.028-1.103), with the rest parameters failed to reach a significant result (Table 2). Besides, neutrophil count significantly correlated with tumor length $(p=0.000)$, portal vein thrombosis $(p=0.036)$, lymph node metastasis $(p=0.032)$, TNM7th stage $(p=0.000)$ and AFP $(p=0.002)$. Since neutrophils count well acknowledged as a prognostic factors in $\mathrm{HCC}$ patients, its association with MDSC level shall be the latent mechanism, which indicated the prognostic value of MDSC.

\section{DISCUSSION}

In this era of immunotherapy, patient selection for clinical trials on novel targets has become a top issue for presenting efficacy $[15,16]$. Myeloid derived suppressor cell (MDSC) has been raised to be a novel target for multiple cancers with dozens of clinical available agents developed $[12,17]$. However, target agents on MDSC has not displayed promising efficacy in major cancer types including HCC. One of the critical reasons shall be less optimal patient selection. HCC is a highly heterogeneous disease [18-20], which makes patients selection more complicated. MDSC testing for patients was not easily to be standard between investigators and institutions [7], thus it was imperative to find clinically feasible parameters that were highly relevant to MDSC level. Then patient selection for MDSC targeted therapy might be facilitated by specific parameters. In the present study, we identified blood neutrophils count were relevant to MDSC level and a strong prognostic factor for HCC patients. Future clinical trials investigating MDSC 
Table 1: Baseline demographic and clinical characteristics of hepatocellular carcinoma patients in testing group and validation group

\begin{tabular}{|c|c|c|c|}
\hline Characteristics & Testing Group $(n=55)$ & Validation Group $(n=245)$ & \\
\hline Gender & & & 0.175 \\
\hline Male & $52(94.5 \%)$ & $213(86.9 \%)$ & \\
\hline Female & $3(5.5 \%)$ & $32(13.1 \%)$ & \\
\hline Age (years) & $56.4(32.0 \sim 79.0)$ & $57.0(19.0-86.0)$ & 0.468 \\
\hline HBsAg & $48(87.3 \%)$ & $214(87.3 \%)$ & 0.999 \\
\hline Liver cirrhosis & $41(74.5 \%)$ & $183(74.7 \%)$ & 0.999 \\
\hline $\operatorname{MDSC}(\%)$ & $10.33(1.43-35.34)$ & & \\
\hline PMN-MDSC(\%) & $6.74(0.60-26.03)$ & & \\
\hline M-MDSC(\%) & $2.79(0.00-13.99)$ & & \\
\hline \multicolumn{4}{|l|}{ Laboratory parameters } \\
\hline International normalized ratio & $1.16(0.95 \sim 4.01)$ & $1.17(0.85-2.53)$ & 0.901 \\
\hline $\operatorname{AFP}(\mathrm{ng} / \mathrm{mL})$ & $274(1.73 \sim 121000)$ & $708.6(1-212620)$ & 0.517 \\
\hline $\operatorname{Albumin}(\mathrm{g} / \mathrm{L})$ & $35.4(24.0 \sim 54.0)$ & $34.6(25.2-51.8)$ & 0.193 \\
\hline ALP (U/L) & $129(50 \sim 1591)$ & $149(33-767)$ & 0.331 \\
\hline Fibrinogen $(\mathrm{g} / \mathrm{L})$ & $2.73(1.11 \sim 5.67)$ & $3.05(0.90-9.40)$ & 0.534 \\
\hline Total bilirubin $(\mu \mathrm{mol} / \mathrm{L})$ & $24.3(9.2 \sim 540)$ & $35.7(5-865)$ & 0.278 \\
\hline $\operatorname{ALT}(\mathrm{U} / \mathrm{L})$ & $51(10 \sim 1284)$ & $55(4-2669)$ & 0.193 \\
\hline Platelets $\left(\times 10^{9} / \mathrm{L}\right)$ & $164(4 \sim 421)$ & $141(4-503)$ & 0.180 \\
\hline Hemoglobin $(\mathrm{g} / \mathrm{L})$ & $126(63 \sim 356)$ & $119(56-177)$ & 0.086 \\
\hline Monocyte $\left(\times 10^{9} / \mathrm{L}\right)$ & $0.58(0.07 \sim 1.42)$ & $0.60(0.02-2.95)$ & 0.121 \\
\hline Neutrophil $\left(\times 10^{9} / \mathrm{L}\right)$ & $4.5(1.4 \sim 16.6)$ & $4.3(0.5-30.6)$ & 0.264 \\
\hline Lymphocyte $\left(\times 10^{9} / \mathrm{L}\right)$ & $1.22(0.20 \sim 6.96)$ & $1.17(0.32-14.0)$ & 0.829 \\
\hline TNM 7th Edition & & & 0.07 \\
\hline I & $13(23.6 \%)$ & $30(12.2 \%)$ & \\
\hline II & $8(14.5 \%)$ & $21(8.6 \%)$ & \\
\hline III & $21(56.4 \%)$ & $140(57.1 \%)$ & \\
\hline IV & $3(5.5 \%)$ & $54(22.0 \%)$ & \\
\hline CLIP score & & & 0.067 \\
\hline 0 & $7(12.7 \%)$ & $11(4.5 \%)$ & \\
\hline 1 & $7(12.7 \%)$ & $23(9.4 \%)$ & \\
\hline 2 & $4(7.3 \%)$ & $39(15.9 \%)$ & \\
\hline 3 & $11(20.0 \%)$ & $41(16.7 \%)$ & \\
\hline 4 & $14(25.5 \%)$ & $60(24.5 \%)$ & \\
\hline 5 & $8(14.5 \%)$ & $62(25.3 \%)$ & \\
\hline 6 & $4(7.3 \%)$ & $9(3.7 \%)$ & \\
\hline
\end{tabular}

Abbreviations: AFP, $\alpha$-fetoprotein; ALP, alkaline phosphatase; ALT, alanineaminotransferase; CLIP, Cancer of the Liver Italian Program. 
Table 2: Multivariate Analysis and Integration into CLIP of the prognostic factors for OS associated with MDSC level among HCC patients

\begin{tabular}{lccc}
\hline \multicolumn{1}{c}{ Characteristics } & $\boldsymbol{P}$ & HR & 95\% CI for HR \\
\hline CLIP & 0.000 & 1.529 & $1.385-1.688$ \\
Neutrophil & 0.000 & 1.065 & $1.028-1.103$ \\
Lymphocyte & 0.895 & 0.988 & $0.825-1.184$ \\
Platelets & 0.454 & 1.001 & $0.999-1.002$ \\
Hemoglobin & 0.413 & 0.997 & $0.991-1.004$ \\
Monocyte & 0.702 & 0.930 & $0.641-1.349$ \\
\hline
\end{tabular}

Abbreviation: CLIP, Cancer of the liver Italian Program scoring system for hepatocelluar carcinoma; OS, overall survival; HCC, hepatocellular carcinoma; HR, hazardsratio; $95 \%$ CI,95\% confidence interval.
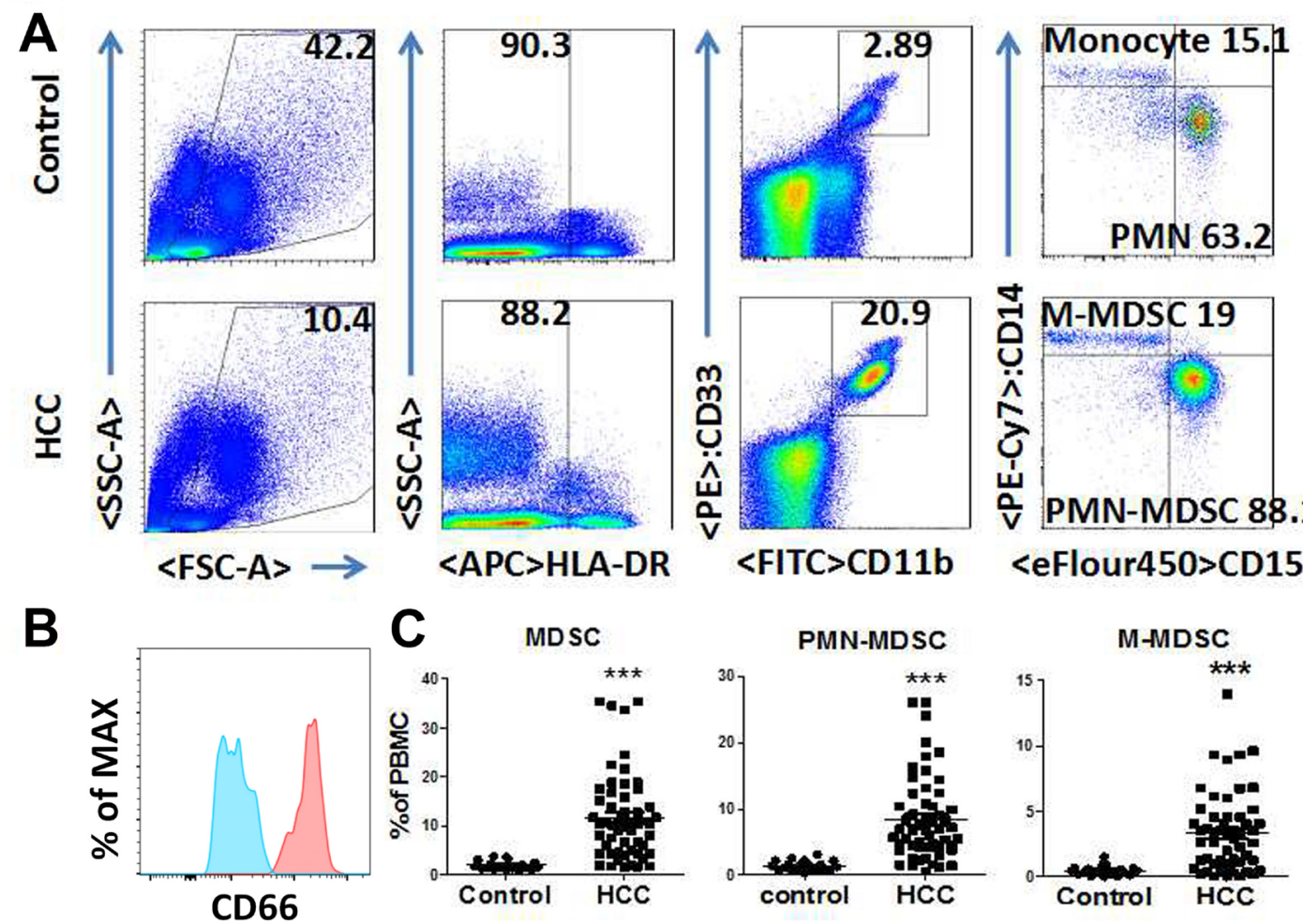

C MDSC
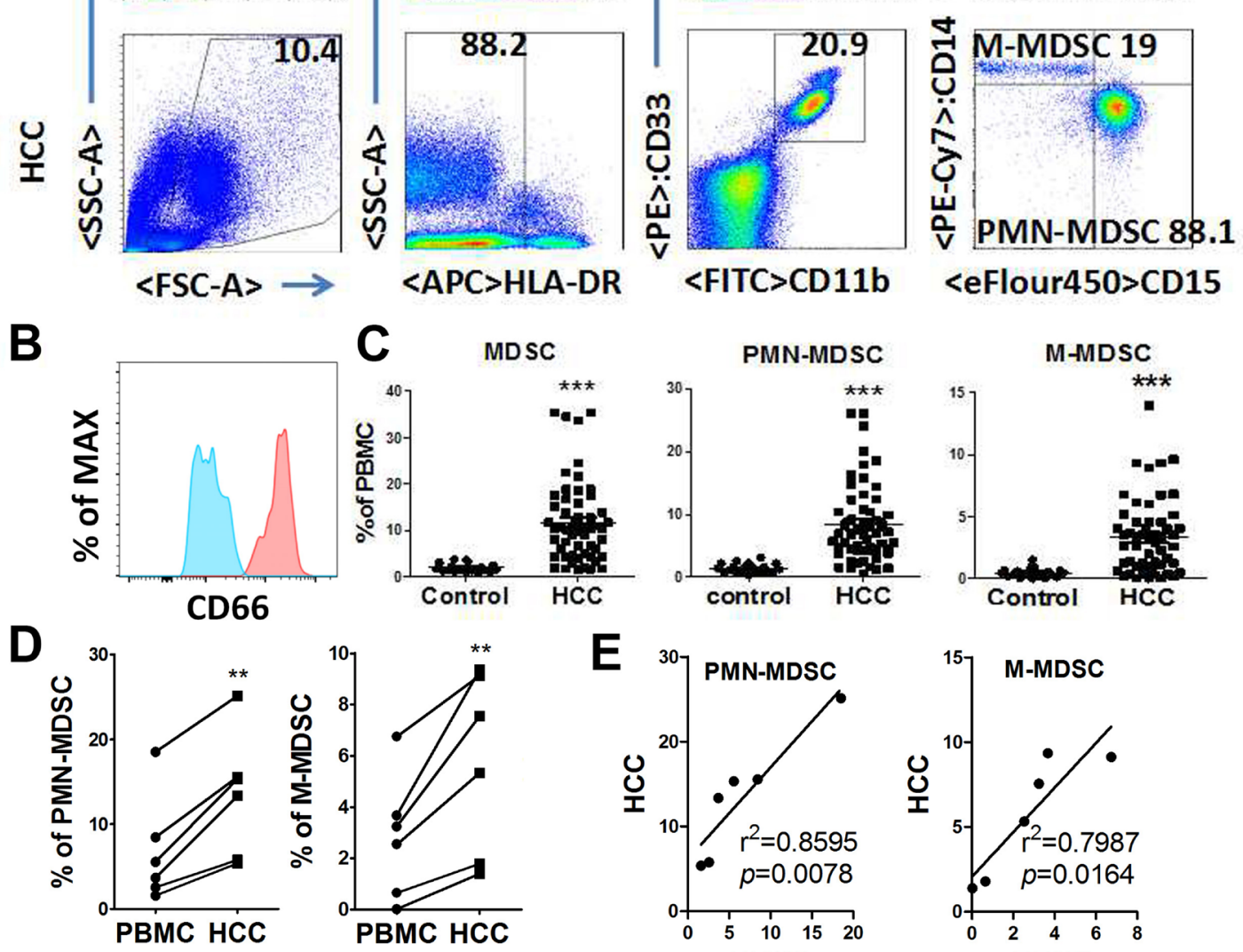

E
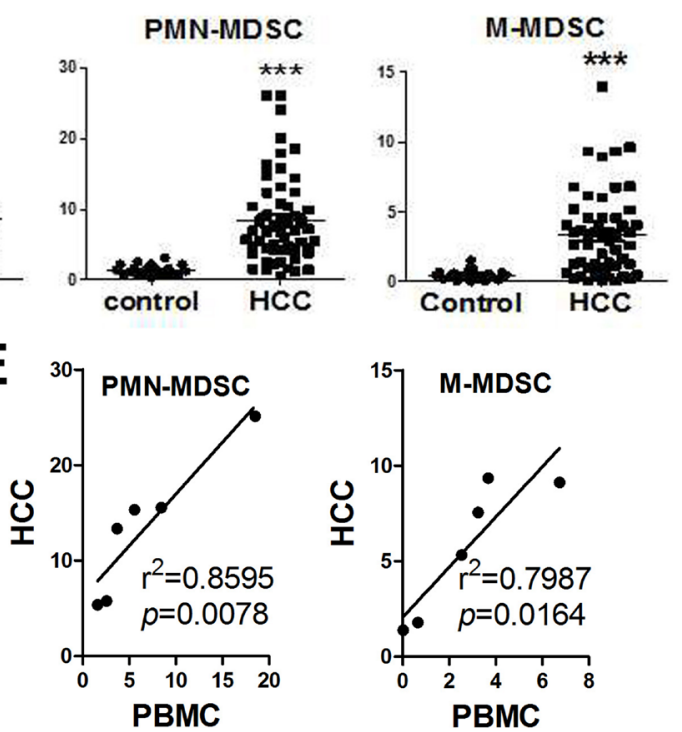

Figure 1: Expansion of PMN-MDSC and M-MDSC in HCC patients. (A) Gating strategy of PMN-MDSC/M-MDSC by flow cytometry analysis. PMN-MDSC was defined as HLA-DR ${ }^{-1 / \mathrm{low}} \mathrm{CD} 11 \mathrm{~b}^{+} \mathrm{CD} 33^{+} \mathrm{CD} 14^{-} \mathrm{CD} 15^{+}$, with M-MDSC defined as HLA-DR ${ }^{-/ l o w}$

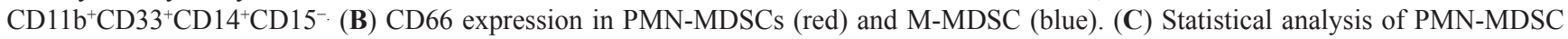
and M-MDSC frequency in the peripheral blood from HCC patients and healthy controls. (D) Comparison of PMN-MDSC/M-MDSC level in PBMC and tumor tissue. (E) Association of PMN-MDSC/M-MDSC level in PBMC and tumor tissue by Linear regression. ${ }^{* *} P<0.01$; *** $P<0.001$. 
targeted therapy might include neutrophil count as one of the criteria.

Our series studies and many reports revealed that neutrophils count was an independent prognostic factor for HCC patients[8]. However, the underlining mechanism was far from identified. Some studies suggested that the neutrophil levels were related to the systematic release of chemokines and ILs, including vascular endothelial growth factor [21], angiopoietin-1 [22] and matrix metalloproteinases-9 [23], which promoted tumor growth and metastasis in HCC. Besides, the presence of neutrophils in the tumor stroma was associated with a poor prognosis[24]. In the present study, we proposed a more reasonable explanation, which was neutrophils count positively associated with MDSC level, which was a well known promoter of malignant diseases.

PMN-MDSC were a subset of neutrophils with similar shape with normal ones [12, 23] and would be designated as neutrophils in routing blood test by automatic blood cell analyzer. Thus, neutrophils consisted of normal neutrophils and PMN-MDSCs. Elevation of
PMN-MDSC had been confirmed in HCC patients by multiple studies [1-3]. The heterogeneity in PMN-MDSC levels among HCC patients decreased the accuracy of the patient selection for MDSCs targeted therapy. In the present study, we revealed that neutrophils count positively related to PMN-MDSC level, which indicated that utilization of neutrophil counts into patient selection might promote the accuracy of picking patients with optimal MDSC level.

Monocyte counts were relevant to M-MDSC levels in our study. M-MDSC presented similar morphology with monocytes $[12,23]$ and automatic blood cell analyzer shall identify it as monocytes. M-MDSC levels were slightly relevant to platelet count, which was a novel finding. Platelet count was considered a prognostic factor for HCC and an indicator of systematic inflammation [10]. The underling interaction between MDSC and platelet needs further investigation.

In summary, the present study firstly identified blood neutrophils counts was a predictor of MDSC level in PBMC for HCC patients. And, patients with higher

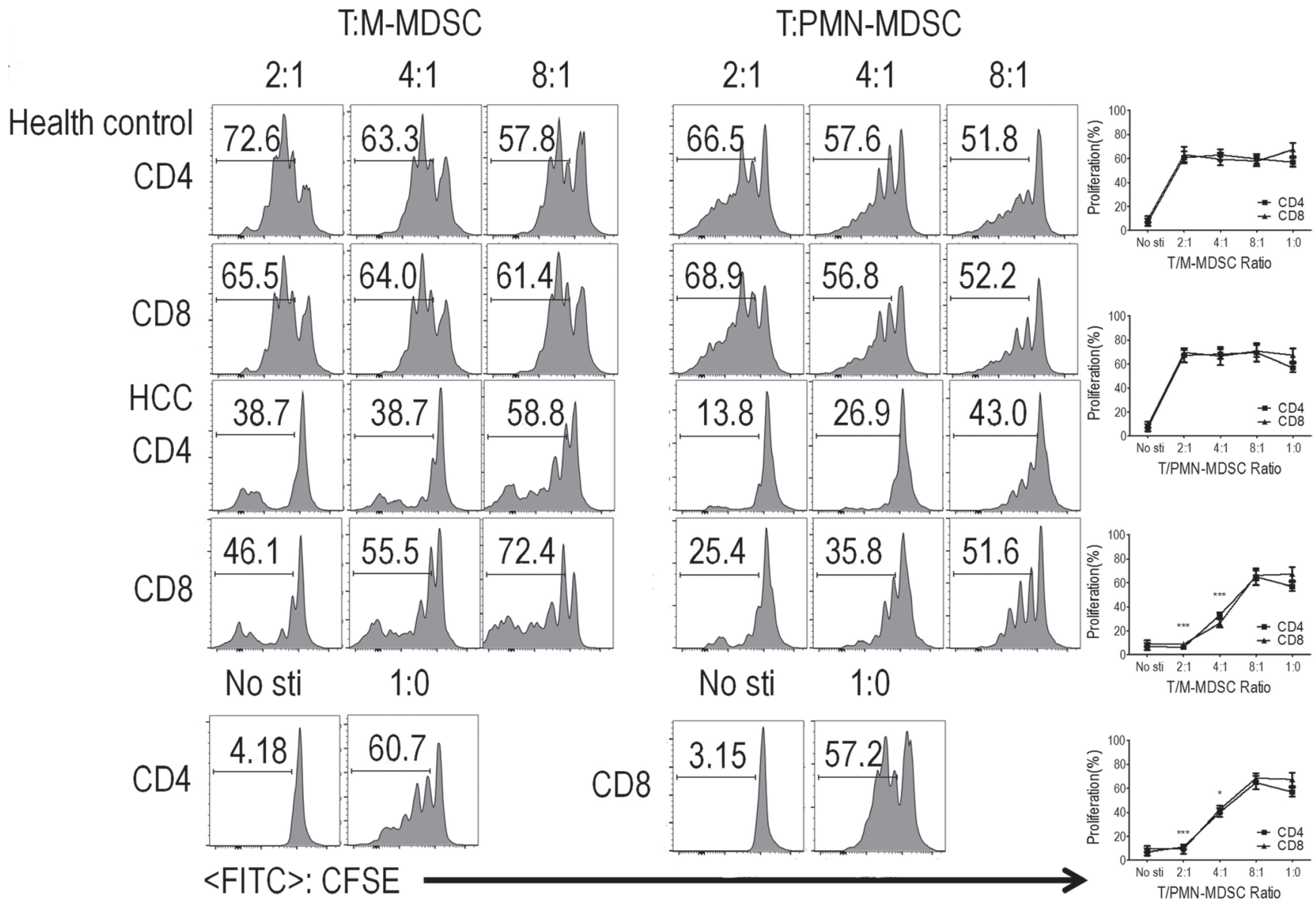

Figure 2: PMN-MDSC and M-MDSC from HCC patients and health donor suppressed $\mathrm{T}$ cell proliferation and activation. $\mathrm{CD} 3+\mathrm{T}$ cells from PBMCs were stimulated with anti-CD3 and anti-CD28, co-cultured with PMN-MDSCs/M-MDSC from the same donors at different ratios for 3 days, and evaluated for CD4+ and CD8+ T cell proliferation by CFSE labeling. Left panels: Representative flow cytometry data from 1 individual. Right panels: Cumulative data. Bottom: Representative flow cytometry data of positive control and negative control. $(n=5)$. $* P<0.05$; *** $P<0.001$. 

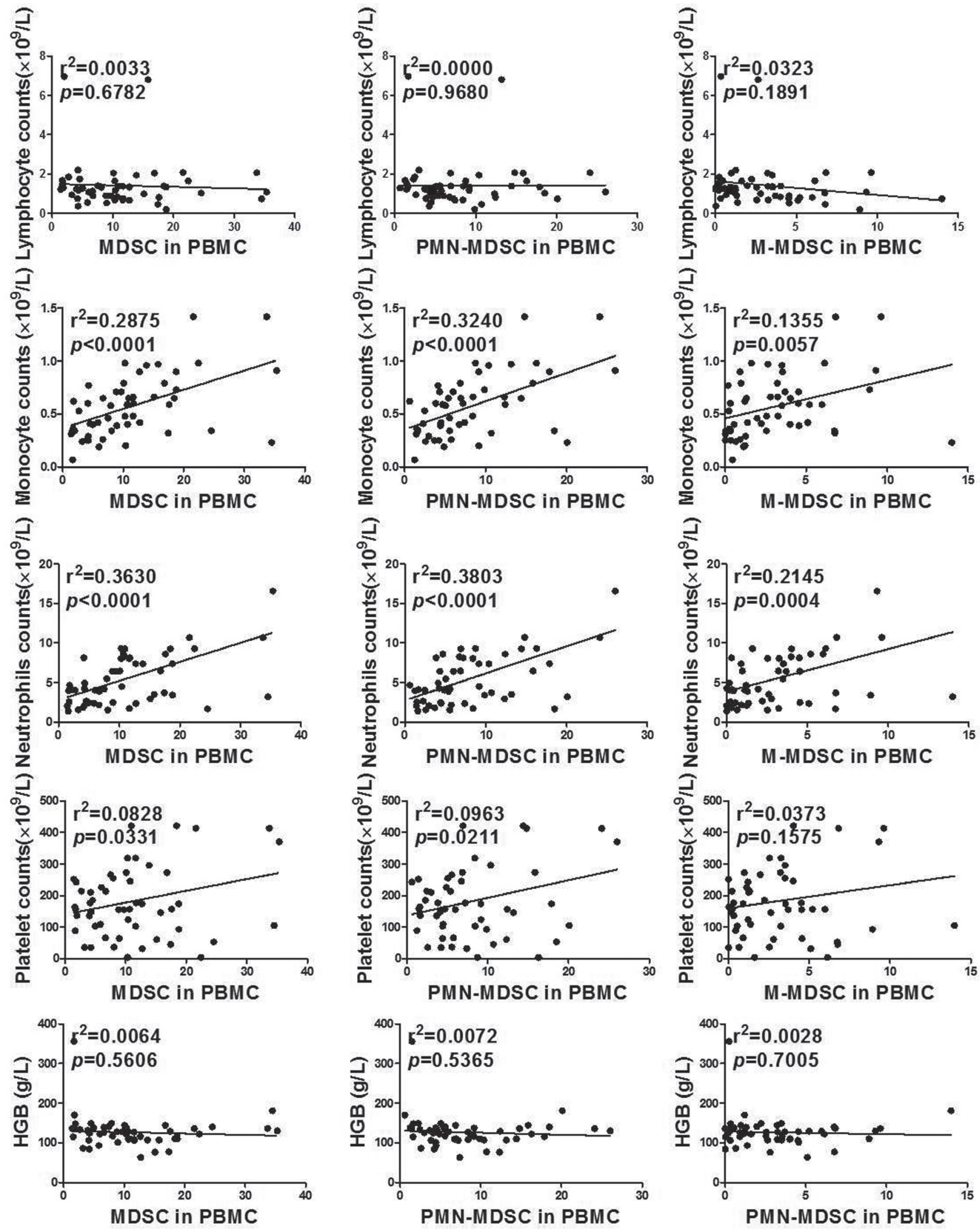

Figure 3: Association between MDSC/PMN-MDSC/M-MDSC and clinical parameters of peripheral blood cell counts by Linear regression. 
neutrophils count level might be the optimal patient subgroup for MDSC targeted therapy.

\section{MATERIALS AND METHODS}

\section{Patients and healthy donors}

During the period between September 2014 and June 2015, we investigated a series of 55 advanced HCC patients who presented to the Third Affiliated Hospital of Sun Yat-sen University, Guangzhou, China (testing group). The diagnosis of HCC was confirmed by pathology or the American Association for the study of liver diseases radiological criteria by either computed tomography (CT) or magnetic resonance imaging (MRI). Age and gender matched healthy controls $(n=20)$ consisted of local volunteers. All patients and healthy control were also screened for serum human immunodeficiency virus (HIV) antibody, hepatitis B surface antigen (HBsAg), hepatitis C virus (HCV) antibody, hepatitis D virus (HDV) antigen and HDV antibody. Patients and healthy controls who were positive for HIV or chronic of hepatitis virus infection except for HBV, and who presented acute infections (including pneumonia, urinary tract infection and et al), who displayed pyrexial (temperature under the axillary is at or over $37.2^{\circ} \mathrm{C}\left(99.0^{\circ} \mathrm{F}\right)$ within 1 week before admission, who were pregnant, patients who received systematic corticosteroids or immnuno-suppressive agents and those younger than 18 years old were excluded from thisstudy. None of the patients received any anti-cancer therapy before collection of PBMCs. This study was approved by the Clinical Ethics ReviewBoard of the Third Affiliated Hospital of Sun Yat-sen University. A written informed consent was obtained from all thepatients at the time of admission.

\section{Mononuclear cells in peripheral blood and tumor tissue isolation and flow cytometric analysis}

Tissues were first digested with the Tumor Dissociation Kit, human AQ56 (Miltenyi), and then red blood cells were lysed. Mononuclear cells were isolated from whole blood or tissue suspension by Ficoll centrifugation and analyzed within 6 hour after blood sampling. The following anti-human antibodies were purchased from eBioscience(San Diego, CA): CD11bfluorescein isothiocyanate (FITC), HLA-DR-APC, CD14-PE-Cy7, CD15-eFluor450, CD33-PE, CD33PerCP-Cyanine5.5, CD66b-PE and their corresponding isotype controls. The cellphenotype was analyzed by flow cytometry on a flow cytometer FACSAria II flow cytometer (BD Bioscience), and data were analyzed with the FlowJo V10.0.7 (FlowJo, OR, USA).

\section{T cell proliferation assay}

$\mathrm{T}$ cell proliferation was illustrated by 5,6-carboxyfluoresceindiacetate, succinimidylester (CFSE) dilution. Purified T cells were labeled with CFSE $(3 \mu \mathrm{M}$; Invitrogen), stimulated with $0.5 \mu \mathrm{g} / \mathrm{ml} 3$-h pre-coated antiCD3 and $0.5 \mu \mathrm{g} / \mathrm{ml}$ anti-CD28 (eBioscience), and cultured alone or co-cultured with autologous PMN-MDSCs or M-MDSC at the indicated ratios for 3 days. The cells were then stained for surface marker expression with CD4-PE or CD8-PE-Cy5 antibodies, and T cell proliferation was analyzed on a flow cytometer. All cultures were conducted in the presence of $20 \mathrm{IU} / \mathrm{ml}$ recombinant human IL-2 (Miltenyi Biotec) in RPMI 1640 (Life Technologies) for $3-4$ days at $37^{\circ} \mathrm{C}$.

\section{Data collection}

Institutional review board approval was obtained from the electronic charts needed to retrieve data regarding the potential prognostic factors, including age, sex, Karnofsky performance status (KPS), pre-therapy laboratory counts of neutrophils, lymphocytes, monocyte, hemoglobin, platelets and parameters included in ChildPugh score and CLIP score and Seventh Edition AJCC tumor-node-metastasis (TNM) staging.

\section{Validation of the clinical parameters related to MDSC level as prognostic factors}

In order to verify the prognostic value of the clinical parameters associated with MDSC level, a series of 245 consecutive HCC patients (validation group) were retrospectively collected and analyzed. The inclusion and exclusion criteria were the same the prospective group. The clinical parameters before anti-cancer treatment were collected and analyzed on their predictive value for OS. All patients underwent regular follow-up in our hospital. OS was calculated from the first day of treatment to the date of death from any cause.

\section{Statistics}

The Kolmogorov-Smirnov test was used to evaluate the normality of distribution. Data were reported as median and range when distribution was not normal. Statistical differences in clinical characteristics between the 2 groups were compared using the $t$-test, the Mann-Whitney test or chi-square test. Group comparison tests were performed using the Wilcoxon rank-sum test. Linear regression was used to find correlation between clinical parameters and the MDSC levels. Multivariate analysis using a Logistic proportional hazards model was used to test for independent significance of all the clinical parameters relevant to MDSC level. The Spearman correlation was 
utilized to determine the association of clinical parameters. For all tests, a $p$ value $<0.05$ was considered statistically significant, and all $p$ values quoted are 2 -sided. Statistical analyses were performed using SPSS v. 20.0 (SPSS Inc., Chicago, IL, USA).

\section{CONFLICTS OF INTEREST}

No conflicts needed to be declared.

\section{FUNDING}

This study was supported by National Key Basic Research Program of China (No. 2012CB524900), Guangdong Innovative Research Team Program (No. 2009010058), Guangdong Province Universities and Colleges Pearl River Scholar Funded Scheme (GDUPS, 2014), Program for the 12th Five-year Plan (No. 2012ZX10001003), National Natural Science Foundation of China (No. 81072397; No.31270921; No. 301500740 and No. 31600710), Natural Science Foundation of Guangdong (No. S2011020006072, No. 2014A030313146 and 2016A030313302), the Fundamental Research Funds for the Central Universities, the Provincial Talents Cultivated by "Thousand-Hundred-Ten" program of Guangdong Province, 111 Project (No. B12003), Innovative Training Program of Sun Yat-sen University (201601073) and Laboratory Open Fund of Sun Yat-sen University (20160117).

\section{REFERENCES}

1. Hu CE, Gan J, Zhang RD, Cheng YR, Huang GJ. Up-regulated myeloid-derived suppressor cell contributes to hepatocellular carcinoma development by impairing dendritic cell function. Scand J Gastroenterol. 2011; 46:156-164.

2. Kalathil S, Lugade AA, Miller A, Iyer R, Thanavala Y. Higher frequencies of GARP $(+)$ CTLA-4(+)Foxp3(+) T regulatory cells and myeloid-derived suppressor cells in hepatocellular carcinoma patients are associated with impaired T-cell functionality. Cancer Res. 2013; 73:2435-2444.

3. Chiu DK, Xu IM, Lai RK, Tse AP, Wei LL, Koh HY, Li LL, Lee D, Lo RC, Wong CM, Ng IO, Wong CC. Hypoxia induces myeloid-derived suppressor cell recruitment to hepatocellular carcinoma through chemokine (C-C motif) ligand 26. Hepatology. 2016; 64:797-813.

4. Wang D, An G, Xie S, Yao Y, Feng G. The clinical and prognostic significance of CD14(+)HLA-DR(-/ low) myeloid-derived suppressor cells in hepatocellular carcinoma patients receiving radiotherapy. Tumour Biol. 2016; 37:10427-10433.

5. Gao XH, Tian L, Wu J, Ma XL, Zhang CY, Zhou Y, Sun YF, Hu B, Qiu SJ, Zhou J, Fan J, Guo W, Yang XR. Circulating CD14+ HLA-DR-/low myeloid-derived suppressor cells predicted early recurrence of hepatocellular carcinoma after surgery. Hepatol Res. 2016.

6. Mizukoshi E, Yamashita T, Arai K, Terashima T, Kitahara M, Nakagawa H, Iida N, Fushimi K, Kaneko S. Myeloid-derived suppressor cells correlate with patient outcomes in hepatic arterial infusion chemotherapy for hepatocellular carcinoma. Cancer Immunol Immunother. 2016; 65:715-725.

7. Grutzner E, Stirner R, Arenz L, Athanasoulia AP, Schrodl K, Berking C, Bogner JR, Draenert R. Kinetics of human myeloid-derived suppressor cells after blood draw. J Transl Med. 2016; 14:2.

8. Li X, Chen ZH, Ma XK, Chen J, Wu DH, Lin Q, Dong M, Wei L, Wang TT, Ruan DY, Lin ZX, Xing YF, Deng Y, et al. Neutrophil-to-lymphocyte ratio acts as a prognostic factor for patients with advanced hepatocellular carcinoma. Tumour Biol. 2014; 35:11057-11063.

9. Lin ZX, Ruan DY, Li Y, Wu DH, Ma XK, Chen J, Chen ZH, Li X, Wang TT, Lin Q, Wen JY, Wu XY. Lymphocyteto-monocyte ratio predicts survival of patients with hepatocellular carcinoma after curative resection. World J Gastroenterol. 2015; 21:10898-10906.

10. Li X, Chen ZH, Xing YF, Wang TT, Wu DH, Wen JY, Chen J, Lin Q, Dong M, Wei L, Ruan DY, Lin ZX, Wu XY, et al. Platelet-to-lymphocyte ratio acts as a prognostic factor for patients with advanced hepatocellular carcinoma. Tumour Biol. 2015; 36:2263-2269.

11. Lin ZH, Li X, Hong YF, Ma XK, Wu DH, Huang M, Chen ZH, Chen J, Dong M, Wei L, Wang TT, Ruan DY, Lin ZX, et al. Alanine aminotransferase to hemoglobin ratio is an indicator for disease progression for hepatocellular carcinoma patients receiving transcatheter arterial chemoembolization. Tumour Biol. 2016; 37:2951-2959.

12. Talmadge JE, Gabrilovich DI. History of myeloid-derived suppressor cells. Nat Rev Cancer. 2013; 13:739-752.

13. Bronte V, Brandau S, Chen SH, Colombo MP, Frey AB, Greten TF, Mandruzzato S, Murray PJ, Ochoa A, OstrandRosenberg S, Rodriguez PC, Sica A, Umansky V, et al. Recommendations for myeloid-derived suppressor cell nomenclature and characterization standards. Nat Commun. 2016; 7:12150.

14. Li X, Dong M, Lin Q, Chen ZH, Ma XK, Xing YF, Wan XB, Wen JY, Wei L, Chen J, Wu XY. Comparison of current staging systems for advanced hepatocellular carcinoma not amendable to locoregional therapy as inclusion criteria for clinical trials. Asia Pac J Clin Oncol. 2013; 9:86-92.

15. Meric-Bernstam F, Brusco L, Shaw K, Horombe C, Kopetz S, Davies MA, Routbort M, Piha-Paul SA, Janku F, Ueno N, Hong D, De Groot J, Ravi V, et al. Feasibility of Large-Scale Genomic Testing to Facilitate Enrollment Onto Genomically Matched Clinical Trials. J Clin Oncol. 2015; 33:2753-2762.

16. Hyman DM, Solit DB, Arcila ME, Cheng DT, Sabbatini P, Baselga J, Berger MF, Ladanyi M. Precision medicine at Memorial Sloan Kettering Cancer Center: clinical next- 
generation sequencing enabling next-generation targeted therapy trials. Drug Discov Today. 2015; 20:1422-1428.

17. Najjar YG, Finke JH. Clinical perspectives on targeting of myeloid derived suppressor cells in the treatment of cancer. Front Oncol. 2013; 3:49.

18. Gao Q, Wang XY, Zhou J, Fan J. Heterogeneity of intermediatestage HCC necessitates personalized management including surgery. Nat Rev Clin Oncol. 2015; 12:10.

19. Nault JC, Villanueva A. Intratumor molecular and phenotypic diversity in hepatocellular carcinoma. Clin Cancer Res. 2015; 21:1786-1788.

20. Jeng KS, Chang CF, Jeng WJ, Sheen IS, Jeng CJ. Heterogeneity of hepatocellular carcinoma contributes to cancer progression. Crit Rev Oncol Hematol. 2015; 94:337-347.

21. Gong Y, Koh DR. Neutrophils promote inflammatory angiogenesis via release of preformed VEGF in an in vivo corneal model. Cell Tissue Res. 2010; 339:437-448.
22. Neagoe PE, Brkovic A, Hajjar F, Sirois MG. Expression and release of angiopoietin-1 from human neutrophils: intracellular mechanisms. Growth Factors. 2009; 27:335-344.

23. Kumar V, Patel S, Tcyganov E, Gabrilovich DI. The Nature of Myeloid-Derived Suppressor Cells in the Tumor Microenvironment. Trends Immunol. 2016; 37:208-220.

24. Zhou SL, Dai Z, Zhou ZJ, Wang XY, Yang GH, Wang Z, Huang XW, Fan J, Zhou J. Overexpression of CXCL5 mediates neutrophil infiltration and indicates poor prognosis for hepatocellular carcinoma. Hepatology. 2012; 56:2242-2254. 